KYUNGPOOK Math. J. 51(2011), 345-352

http://dx.doi.org/10.5666/KMJ.2011.51.3.345

\title{
On Paranormed Type Fuzzy Real Valued Class of Sequences ${ }_{2} \ell^{F}(p)$
}

\author{
Mausumi Sen* and Santanu Roy \\ Department of Mathematics, National Institute of Tecgnology, Silchar, 788010, \\ India \\ e-mail : sen_mausumi@rediffmail.com and santanuroy@yahoo.in
}

ABStract. In this article we introduce the fuzzy real valued double sequence spaces ${ }_{2} \ell^{F}(p)$ where $p=\left(p_{n k}\right)$ is a double sequence of bounded strictly positive numbers. We study their different properties like completeness, solidness, symmetricity, convergence free etc. We prove some inclusion results also.

\section{Introduction}

The concept of fuzzy sets was introduced by Zadeh [19] in 1965. The potential of the notion of fuzzy set was realized by different scientific groups and many researchers. The concept of fuzziness has been applied in various fields such as cybernetics, artificial intelligence, expert system and fuzzy control, pattern recognition, operation research, decision making, image analysis, projectiles, probability theory, agriculture, weather forecasting, etc.

Using the notion of fuzzy real numbers, different types of fuzzy real-valued sequence spaces have been introduced and studied by Nanda [5], Nuray and Savas [6], Tripathy and Dutta ([10], [11], [12]), Tripathy and Borgohain [18], Tripathy and Baruah ([8], [9]), Tripathy and Sarma [15], Choudhary and Tripathy [2] and many others. The initial works on double sequences of real or complex terms is found in Bromwich [1]. The notion of regular convergence of double sequences of real or complex terms is introduced by Hardy [2]. Tripathy and Sarma ([13], [14]) studied different types of double sequence spaces, while Tripathy and Dutta ([10], [11], [12]) introduced and investigated different types of double sequence spaces.

The notion of paranormed sequence spaces is pity old. Different classes of paranormed sequence spaces have been introduced and studied by Choudhary and Tripathy [2], Tripathy and Sen[16], Tripathy[7]. Tripathy and Sen [17] have characterized some matrix classes involving some paranormed sequence spaces, those unify

* Corresponding Author.

Received September 13, 2010; revised December 22, 2010; accepted January 20, 2011.

2010 Mathematics Subject Classification: 40A05, 40A30, 40D25.

Key words and phrases: Fuzzy real valued double sequence, Solid, Monotone, Symmetric, Convergence free, Sequence algebra. 
and generalize several existing results. In this article we shall investigate the class of double sequences ${ }_{2} \ell^{F}(p)$ of paranormed type.

A fuzzy real number $X$ is a fuzzy set on $R$, ie. a mapping $X: R \rightarrow L(=[0,1])$ associating each real number $\mathrm{t}$ with its grade of membership $X(t)$.

Every real number $\mathrm{r}$ can be expressed as a fuzzy real number $\bar{r}$ as follows :

$$
\bar{r}(t)= \begin{cases}1 & \text { if } \mathrm{t}=\mathrm{r} \\ 0 & \text { otherwise. }\end{cases}
$$

The $\alpha$ - level set of a fuzzy real number $X, 0<\alpha \leq 1$ denoted by $[X]^{\alpha}$ is defined as $[X]^{\alpha}=\{t \in R: X(t) \geq \alpha\}$. A fuzzy real number $X$ is called convex if $X(t) \geq$ $X(s) \wedge X(r)=\min (X(s), X(r))$, where $s<t<r$. If there exists $t_{0} \in R$ such that $X\left(t_{0}\right)=1$, then the fuzzy real number $X$ is called normal. A fuzzy real number $X$ is said to be upper semi-continuous if for each $\epsilon>0, X^{-1}([0, a+\epsilon))$, for all $a \in L$ is open in the usual topology of $R$. The set of all upper semi continuous, normal, convex fuzzy number is denoted by $L(R)$. A fuzzy real number $\eta$ is said to be non-negative if $\eta(t)=0$ for all $t<0$.

The absolute value of $X \in L(R)$ is defined as (one may refer to Kaleva and Seikkla $[4])$

$$
|X(t)|= \begin{cases}\max \{X(t), X(-t)\} & \text { for } t \geq 0 \\ 0 & \text { otherwise. }\end{cases}
$$

Let $D$ be the set of all closed bounded intervals $X=\left[\mathrm{X}^{L}, \mathrm{X}^{R}\right]$. Then $X \leq Y$ if and only if $X^{L} \leq Y^{L}$ and $X^{R} \leq Y^{R}$.

Also $d(X, \bar{Y})=\max \left(\left|X^{\bar{L}}-Y^{L}\right|,\left|X^{R}-Y^{R}\right|\right)$

Then $(D, d)$ is a complete metric space and $\leq$ is a partial order on $D$.

Let $\bar{d}: L(R) \times L(R) \rightarrow R$ be defined by

$$
\bar{d}(X, Y)=\sup _{0 \leq \alpha \leq 1} d\left([X]^{\alpha},[Y]^{\alpha}\right), \text { for } X, Y \in L(R) .
$$

Then $\bar{d}$ defines a metric on $L(R)$.

\section{Definitions and background}

Throughout $N, R$ denote the sets of natural and real numbers respectively. Throughout the article a fuzzy real valued double sequence is denoted by $\left\langle X_{n k}\right\rangle$ i.e. a double infinite array of fuzzy real numbers $X_{n k}$ for all $n, k \in N$.

Nanda introduced and studied fuzzy real-valued double sequence space $\ell_{p}^{F}$, for $1 \leq p<\infty$. Nuray and Savas have studied some of the properties of the space $\ell(p)^{F}$.

A fuzzy real valued double sequence space $E^{F}$ is said to be solid if $\left\langle Y_{n k}\right\rangle \in E^{F}$, whenever $\left|Y_{n k}\right| \leq\left|X_{n k}\right|$ for all $n, k \in N$ and $\left\langle X_{n k}\right\rangle \in E^{F}$. A fuzzy real valued double sequence $E^{F}$ is said to be monotone if $E^{F}$ contains the canonical pre-image of all its step spaces. A fuzzy real valued double sequence $E^{F}$ is said to be symmetric if $S(X) \subset E^{F}$, for all $X \in E^{F}$, where $S(X)$ denotes the set of all permutations of 
the elements of $X=\left\langle X_{n k}\right\rangle$. A fuzzy real valued double sequence $E^{F}$ is said to be sequence algebra if $\left\langle X_{n k} \otimes Y_{n k}\right\rangle \in E^{F}$, whenever $\left\langle X_{n k}\right\rangle,\left\langle Y_{n k}\right\rangle \in E^{F}$. A fuzzy real valued double sequence $E^{F}$ is said to be convergence free if $\left\langle Y_{n k}\right\rangle \in E^{F}$, whenever $\left\langle X_{n k}\right\rangle \in E^{F}$ and $X_{n k}=\overline{0}$ implies $Y_{n k}=\overline{0}$.

Throughout $p=\left(p_{n k}\right)$ is a double sequence of bounded strictly positive numbers. We define the following fuzzy real valued double sequence space:

$$
{ }_{2} \ell^{F}(p)=\left\{X=\left\langle X_{n k}\right\rangle: \sum_{n=1}^{\infty} \sum_{k=1}^{\infty}\left[\bar{d}\left(X_{n k}, \overline{0}\right)\right]^{p_{n k}}<\infty\right\} .
$$

Lemma 1. If a sequence space $E^{F}$ is solid, then it is monotone.

\section{Main results}

Theorem 1. The space ${ }_{2} \ell^{F}(p)$ is a complete metric space with respect to the metric $\rho$ defined by

$$
\rho(X, Y)=\left(\sum_{n=1}^{\infty} \sum_{k=1}^{\infty}\left[\bar{d}\left(X_{n k}, y_{n k}\right)\right]^{p_{n k}}\right)^{\frac{1}{M}}, \text { where } M=\max \left(1, \sup _{n k} p_{n k}\right) .
$$

Proof. Let $\left(X^{(i)}\right)$ be a Cauchy sequence in ${ }_{2} \ell^{F}(p)$, where $X^{(i)}=\left\langle X_{n k}^{(i)}\right\rangle$. Then for a given $\epsilon>0$, there exists $n_{0} \in N$, such that $\left.\rho\left(X^{(i)}, X^{(j)}\right)\right)<\epsilon$, for all $i, j \geq n_{0}$.

$$
\begin{aligned}
& \Rightarrow\left(\sum_{n=1}^{\infty} \sum_{k=1}^{\infty}\left[\bar{d}\left(X_{n k}^{(i)}, X_{n k}^{(j)}\right]^{p_{n k}}\right)^{\frac{1}{M}}<\epsilon, \text { for all } i, j \geq n_{0}\right. \\
& \Rightarrow\left(\sum _ { n = 1 } ^ { \infty } \sum _ { k = 1 } ^ { \infty } \left[\bar{d}\left(\left(X_{n k}^{(i)}, X_{n k}^{(j)}\right]^{p_{n k}}\right)<\epsilon^{M}, \text { for all } i, j \geq n_{0}\right.\right. \\
& \Rightarrow \bar{d}\left(X_{n k}^{(i)}, X_{n k}^{(j)}\right)<\epsilon, \text { for all } i, j \geq n_{0}, \text { for all } n, k \in N . \\
& \Rightarrow\left\langle X_{n k}^{(i)}\right\rangle_{i=1}^{\infty} \text { is a Cauchy sequence in } L(R) \text { for each } n, k \in N .
\end{aligned}
$$

Since $L(R)$ is complete, so $\left\langle X_{n k}^{(i)}\right\rangle_{i=1}^{\infty}$ is convergent for each $n, k \in N$.

Let

$$
\left.\lim _{i} X_{n k}^{(i)}\right)=X_{n k}
$$

for each $n, k \in N$ and $X=\left\langle X_{n k}\right\rangle$. Taking limit as $j \rightarrow \infty$ in equation (1), we have $\rho\left(X^{(i)}, X\right)<\epsilon$, for all $i \geq n_{0}$.

Now for all $i \geq n_{0}$

$$
\rho(X, \overline{0}) \leq \rho\left(X, X^{(i)}\right)+\rho\left(X^{(i)}, \overline{0}\right) \leq \epsilon+K<\infty .
$$


Thus $X \in_{2} \ell^{F}(p)$ and so ${ }_{2} \ell^{F}(p)$ is complete. The proof is completed.

Theorem 2. The space ${ }_{2} \ell^{F}(p)$ is solid as well as monotone.

Proof. Let $\left\langle X_{n k}\right\rangle \in_{2} \ell^{F}(p)$ and $\left\langle Y_{n k}\right\rangle$ be a fuzzy real valued double sequence such that $\left|Y_{n k}\right| \leq\left|X_{n k}\right|$ for all $n, k \in N$.

Then

$$
\sum_{n=1}^{\infty} \sum_{k=1}^{\infty}\left[\bar{d}\left(X_{n k}, \overline{0}\right)\right]^{p_{n k}}<\infty
$$

Now

$$
\sum_{n=1}^{\infty} \sum_{k=1}^{\infty}\left[\bar{d}\left(Y_{n k}, \overline{0}\right)\right]^{p_{n k}} \leq \sum_{n=1}^{\infty} \sum_{k=1}^{\infty}\left[\bar{d}\left(X_{n k}, \overline{0}\right]^{p_{n k}}<\infty .\right.
$$

Thus $\left\langle Y_{n k}\right\rangle \in_{2} \ell^{F}(p)$ and so ${ }_{2} \ell^{F}(p)$ is solid.

Also by Lemma 1 , it follows that the space ${ }_{2} \ell^{F}(p)$ is monotone. The proof is completed.

Theorem 3. The space ${ }_{2} \ell^{F}(p)$ is a sequence algebra.

Proof. Let $\left\langle X_{n k}\right\rangle,\left\langle Y_{n k}\right\rangle \in \in_{2} \ell^{F}(p)$.

Then

$$
\left.\sum_{n=1}^{\infty} \sum_{k=1}^{\infty}\left[\bar{d}\left(X_{n k} \otimes Y_{n k}, \overline{0}\right)\right]^{p_{n k}} \leq \sum_{n=1}^{\infty} \sum_{k=1}^{\infty} \bar{d}\left(X_{n k}, \overline{0}\right)\right]^{p_{n k}} \sum_{n=1}^{\infty} \sum_{k=1}^{\infty}\left[\bar{d}\left(Y_{n k}, \overline{0}\right)\right]^{p_{n k}}<\infty .
$$

Thus $\left\langle X_{n k} \otimes Y_{n k}\right\rangle \in \in_{2} \ell^{F}(p)$ and so ${ }_{2} \ell^{F}(p)$ is a sequence algebra. The proof is completed.

Theorem 4. The space ${ }_{2} \ell^{F}(p)$ is not convergence free in general.

Proof. The result follows from the following example.

Example. Let

$$
p_{n k}= \begin{cases}2 & \text { if } n=k \\ \frac{1}{n k} & n \neq k\end{cases}
$$

We consider the sequence $\left\langle X_{n k}\right\rangle$ defined as follows:

$$
X_{n n}(t)= \begin{cases}n^{2} t+1 & \text { for }-\frac{1}{n^{2}} \leq t \leq 0 \\ 1-n^{2} t & \text { for } 0 \leq t \leq \frac{1}{n^{2}} \\ 0 & \text { otherwise. }\end{cases}
$$

and $X_{n k}=\overline{0}$ for $n \neq k$.

Then

$$
\sum_{n=1}^{\infty} \sum_{k=1}^{\infty}\left[\bar{d}\left(X_{n k}, \overline{0}\right)\right]^{p_{n k}}=\sum_{n=1}^{\infty}\left(\frac{1}{n^{2}}\right)^{2}=\sum_{n=1}^{\infty} \frac{1}{n^{4}}<\infty .
$$

Then $\left\langle X_{n k}\right\rangle \in_{2} \ell^{F}(p)$.

Let us consider the sequence $\left\langle Y_{n k}\right\rangle$ defined as follows: 


$$
Y_{n n}(t)= \begin{cases}\frac{1}{2}\left(1+\frac{t}{n^{2}}\right) & \text { for }-n^{2} \leq t \leq n^{2} \\ 2-\frac{t}{n^{2}} & \text { for } n^{2} \leq t \leq 2 n^{2} \\ 0 & \text { otherwise. }\end{cases}
$$

and $Y_{n k}=\overline{0}$ for $n \neq k$.

Then

$$
\sum_{n=1}^{\infty} \sum_{k=1}^{\infty}\left[\bar{d}\left(Y_{n k}, \overline{0}\right)\right]^{p_{n k}}=\sum_{n=1}^{\infty}\left(2 n^{2}\right)^{2}=\infty .
$$

Then $\left\langle Y_{n k}\right\rangle \notin_{2} \ell^{F}(p)$. Hence the space ${ }_{2} \ell^{F}(p)$ is not convergence free. The proof is completed.

Theorem 5. The space ${ }_{2} \ell^{F}(p)$ is not symmetric.

Proof. The result follows from the following example.

Example. Let

$$
p_{n k}= \begin{cases}2 & \text { if } n=k \\ 1 & \text { otherwise. }\end{cases}
$$

We consider the sequence $\left\langle X_{n k}\right\rangle$ defined by :

$$
X_{n n}(t)= \begin{cases}1+\sqrt{2} n t, & \text { for }-\frac{1}{\sqrt{2} n} \leq t \leq 0 \\ 1-\sqrt{2} n t, & \text { for } 0 \leq t \leq \frac{1}{\sqrt{2} n} \\ 0 & \text { otherwise. }\end{cases}
$$

and $X_{n k}=\overline{0}$ for $n \neq k$.

Then

$$
\sum_{n=1}^{\infty} \sum_{k=1}^{\infty}\left[\bar{d}\left(X_{n k}, \overline{0}\right)\right]^{p_{n k}}=\sum_{n=1}^{\infty}\left(\frac{1}{\sqrt{2} n}\right)^{2}=\sum_{n=1}^{\infty} \frac{1}{2 n^{2}}<\infty .
$$

Thus $\left\langle X_{n k}\right\rangle \in_{2} \ell^{F}(p)$.

Let us consider the rearrangement $\left\langle Y_{n k}\right\rangle$ of $\left\langle X_{n k}\right\rangle$ defined as follows :

$\left\langle Y_{n k}\right\rangle=\left(\begin{array}{cccc}X_{11} & X_{12} & X_{13} & \cdots \\ X_{22} & X_{21} & X_{23} & \cdots \\ X_{33} & X_{32} & X_{31} & \cdots \\ \vdots & \vdots & \vdots & \ddots\end{array}\right)$

i.e.

$$
Y_{n k}= \begin{cases}X_{n n}, & \text { if } k=1 \text { for all } n \in N \\ X_{n 1}, & \text { if } k=n, \\ X_{n k}, & \text { otherwise. }\end{cases}
$$

Then

$$
\sum_{n=1}^{\infty} \sum_{k=1}^{\infty}\left[\bar{d}\left(Y_{n k}, \overline{0}\right)\right]^{p_{n k}}=\left(\frac{1}{\sqrt{2} 1}\right)^{2}+\sum_{n=1}^{\infty} \frac{1}{\sqrt{2} n}=\infty .
$$


Hence $\left\langle Y_{n k}\right\rangle \notin_{2} \ell^{F}(p)$ and so the space ${ }_{2} \ell^{F}(p)$ is not symmetric. The proof is completed.

Theorem 6. If

$$
0<p_{n k}<q_{n k} \leq \sup _{n k} q_{n k},
$$

then ${ }_{2} \ell^{F}(p) \subset_{2} \ell^{F}(q)$ and the inclusion is proper.

Proof. Let $\left\langle X_{n k}\right\rangle \in_{2} \ell^{F}(p)$.

Then

$$
\sum_{n=1}^{\infty} \sum_{k=1}^{\infty}\left[\bar{d}\left(X_{n k}, \overline{0}\right)\right]^{p_{n k}}<\infty .
$$

Then there exists $n_{0}, k_{0} \in N$ such that $\left[\bar{d}\left(X_{n k}, \overline{0}\right)\right]^{p_{n k}}<1$ for either $n \geq n_{0}$ or $k \geq k_{0}$ or both.

$$
\begin{gathered}
\Rightarrow\left[\bar{d}\left(X_{n k}, \overline{0}\right)\right]^{q_{n k}}<\left[\bar{d}\left(X_{n k}, \overline{0}\right)\right]^{p_{n k}} \text { for all } n \geq n_{0} \text { or } k \geq k_{0} . \\
\Rightarrow \sum_{n=1}^{\infty} \sum_{k=1}^{\infty}\left[\bar{d}\left(X_{n k}, \overline{0}\right)\right]^{q_{n k}}<\infty .
\end{gathered}
$$

Thus $\left\langle X_{n k}\right\rangle \in_{2} \ell^{F}(q)$. The inclusion is strict and it follows from the following example.

\section{Example.}

We consider the sequence $\left\langle X_{n k}\right\rangle$ defined as follows :

$$
X_{n n}(t)= \begin{cases}1+\sqrt{3} n t & \text { for }-\frac{1}{\sqrt{3} n} \leq t \leq 0 \\ 1-\sqrt{3} n t & \text { for } 0 \leq t \leq \frac{1}{\sqrt{3} n} \\ 0 & \text { otherwise }\end{cases}
$$

and $X_{n k}=\overline{0}$ for $n \neq k$.

Let

$$
q_{n k}= \begin{cases}2+\frac{1}{n} & \text { if for } n=k \\ 3 & \text { for } n \neq k\end{cases}
$$

and

$$
p_{n k}= \begin{cases}1 & \text { if for } n=k \\ 2 & \text { for } n \neq k\end{cases}
$$

Then

$$
\sum_{n=1}^{\infty} \sum_{k=1}^{\infty}\left[\bar{d}\left(X_{n k}, \overline{0}\right)\right]^{q_{n k}}=\sum_{n=1}^{\infty}\left(\frac{1}{\sqrt{3} n}\right)^{2+\frac{1}{n}}<\infty .
$$

and so $\left\langle X_{n k}\right\rangle \in_{2} \ell^{F}(q)$.

But

$$
\sum_{n=1}^{\infty} \sum_{k=1}^{\infty}\left[\bar{d}\left(X_{n k}, \overline{0}\right)\right]^{p_{n k}}=\sum_{n=1}^{\infty}\left(\frac{1}{\sqrt{3} n}\right)=\infty .
$$


and so $\left\langle X_{n k}\right\rangle \notin_{2} \ell^{F}(p)$. The proof is completed.

Acknowledgement. The authors thank the referee for his valuable comments and suggestions.

\section{References}

[1] T. J. I'A Bromaich, An Introduction to the Theory of Infinite Series, Macmillan \& Co. Ltd., New York, 1965.

[2] B. Choudhury, and B. C. Tripathy, On fuzzy real valued $\ell(p)^{F}$ sequence spaces, Proc International Conference, held at Salt Lake city, USA, during July 21-25(2005), 184190.

[3] G. H. Hardy, On the convergence of certain multiple series, Proc. Camb Phil. Soc., 19(1917), 86-95.

[4] O. Kaleva and O. Kaleva, On fuzzy metric spaces, Fuzzy Sets and Systems, 12(1984), 215-229.

[5] S. Nanda, On sequences of fuzzy numbers, Fuzzy Sets and Systems, 33(1989), 123-126.

[6] F. Nuray and E. Savas, Statistical convergence of sequences of fuzzy real numbers, Math. Slovaca, 45(3)(1995), 269-273.

[7] B. C. Tripathy, On generalized difference paranormed statistically convergent sequences, Indian Journal of Pure and Applied Mathematics, 35(5)(2004), 655-663.

[8] B. C. Tripathy and A. Baruah, New type of difference sequence spaces of fuzzy real numbers, Mathematical Modelling and Analysis, 14(3)(2009), 391-397.

[9] B. C. Tripathy and A. Baruah, Nörlund and Riesz mean of sequences of fuzzy real numbers, Applied Mathematics Letters, 23(2010), 651-655.

[10] B. C. Tripathy and A. J. Dutta, Bounded variation double sequence space of fuzzy real numbers, Computers \& Mathematics with Applications. 9(2)(2010), 1031-1037.

[11] B. C. Tripathy and A. J. Dutta, On fuzzy real valued double sequence spaces, Soochow jour. Of Math., 32(4)(2006), 509-520.

[12] B. C. Tripathy and A. J. Dutta, On fuzzy real valued double sequence spaces ${ }_{2} \ell_{F}^{p}$, Mathematical and Computer, 46(2007), 1294-1299.

[13] B. C. Tripathy and B. Sarma, Statistically convergent difference double sequence spaces, Acta Mathematica Sinica, 24(5)(2008), 737-742.

[14] B. C. Tripathy and B. Sarma, Vector valued double sequence spaces defined by Orlicz function, Mathematica Slovaca, 59(6)(2009), 767-776.

[15] B. C. Tripathy and B. Sarma, Sequence spaces of fuzzy real numbers defined by Orlicz functions, Mathematica Slovaca, 58(5)(2008), 621-628.

[16] B. C. Tripathy and M. Sen, On generalized statistically convergent sequences, Indian Journal of Pure and Applied Mathematics, 32(11)(2001), 1689-1694. 
[17] B. C. Tripathy and M. Sen, Characterization of some matrix classes involving paranormed sequence spaces, Tamkang Journal of Mathematics, 37(2)(2006), 155-162.

[18] B. C. Tripathy, and S. Borgohain, The sequence space $m\left(M, \phi, \Delta_{m}^{n}, p\right)^{F}$, Mathematical Modelling and Analysis, 13(4)(2008), 577-586.

[19] L. A. Zadeh, Fuzzy sets,, Information and Control, 8(1965), 338-353. 